\title{
Diversity of Chloroplast DNA in Various Mandarins (Citrus spp.) and Other Citrus Demonstrated by CAPS Analysis
}

\author{
Masashi Yamamoto $^{1 *}$, Yui Tsuchimochi ${ }^{1}$, Takanori Ninomiya ${ }^{1}$, Takanori Koga ${ }^{1}$, \\ Akira Kitajima $^{2}$, Atsu Yamasaki ${ }^{3 * *}$, Sayuri Inafuku-Teramoto ${ }^{4}$, Xuehu Yang ${ }^{5 * * *}$, \\ Xiaoling Yang ${ }^{6}$, Guangyan Zhong ${ }^{7 * * * *}$, Nasril Nasir ${ }^{8}$, Tatsuya Kubo ${ }^{1}$ \\ and Shigeto Tominaga ${ }^{1}$ \\ ${ }^{1}$ Faculty of Agriculture, Kagoshima University, Kagoshima 890-0065, Japan \\ ${ }^{2}$ Experimantal Farm, Graduate School of Agriculture, Kyoto University, Takatsuki 569-0096, Japan \\ ${ }^{3}$ Okitsu Citrus Research Station, NARO Institute of Fruit Tree Science, Shizuoka 424-0292, Japan \\ ${ }^{4}$ Faculty of Agriculture, University of The Ryukyus, Nishihara, Okinawa 903-0213, Japan \\ ${ }^{5}$ United Graduate School of Agricultural Sciences, Kagoshima University, Kagoshima 890-0065, Japan \\ ${ }^{6}$ School of Live Science \& Technology, Tongji University, Shanghai 200092, China \\ ${ }^{7}$ Citrus Research Institute, Southwest University, Chongqing 400712, China \\ ${ }^{8}$ Faculty of Mathematics and Natural Science, Andalas University, Padang 25163, Indonesia
}

The diversity of chloroplast DNA (cpDNA) of citrus was revealed by means of a cleaved amplified polymorphic sequence (CAPS) using 97 Citrus, including many local accessions from Japan, China, and Indonesia, four Fortunella, and two Poncirus. All accessions were classified into seven types based on the results of polymorphic bands in all primer/enzyme combinations. The seven types were identified as follows: Type 1 (38 accessions): C. macroptera, C. hystrix, C. aurantifolia, C. medica, C. limon, C. grandis, C. aurantium, C. myrtifolia, C. bergamia, C. rokugatsu, C. sinensis, C. sphaerocarpa, C. nobilis (Kunenbo), C. keraji, C. oto, C. tarogayo, C. suavissima, mandarins of Yunnan and Guangxi, China, miscellaneous of Yunnan, China, $C$. madurensis, and Fortunella; Type 2 (one accession): C. latipes; Type 3 (two accessions): C. ichangensis and $C$.junos; Type 4 (six accessions): C. tachibana and C.depressa; Type 5 (18 accessions): C.jambhiri, C.tankan, C. sunki, C. reshni, C.depressa, mandarins of Kagoshima, Japan and Yunnan and Guangxi, China; Type 6 (36 accessions): C. nobilis (King), C. unshiu, C. reticulata, C. genshokan, C.clementina, C. succosa, C. suhuensis, C. tardiferax, C.erythora, C. kinokuni, C. oleocarpa, C. leiocarpa, mandarins of Kagoshima, Japan, Guangxi, Zejiang, Yunnan and Guangdone, China, and mandarins of West Sumatra, Indonesia; Type 7 (Two accessions): Poncirus. The differentiation of cpDNA in citrus was discussed in accordance with the results.

Key Words: cpDNA, cytoplasmic genome, differentiation, evolution, polymorphism.

\section{Introduction}

Citrus is one of the most important fruit crops globally. It is cultivated between the latitudes of $40^{\circ}$ north and

\footnotetext{
Received; June 14, 2012. Accepted; January 3, 2013.

* Corresponding author (E-mail: yamasa@agri.kagoshima-u.ac.jp).

** Present address: Grape and Persimmon Research Station, NARO Institute of Fruit Tree Science.

*** Present address: Yunnan Agricultural University.

**** Present address: Institute of Fruit Tree Research, Guangdong Academy of Agricultural Science.
}

south, and the main growing area is the sub-tropical region. There are various accessions/cultivars adapted to different areas with great diversity.

Despite its economic importance, Citrus taxonomy has been difficult to characterize due to wide crosscompatibility, polyembryony (apomixis), the high frequency of bud mutation and a long history of cultivation; however, recent DNA analysis revealed that citron (C. medica), pummelo (C. grandis, synonym: C. maxima), and mandarin (C. reticulata) are the basic species of Citrus. Other species, such as sweet orange (C. sinensis), grapefruit (C.paradisi), and lemon 
(C. limon), are of hybrid origin (Barkley et al., 2006; Federici et al., 1998; Nicolosi et al., 2000; Pang et al., 2007). In addition, several studies have suggested that genetic variations within the mandarin are larger than those in citron and pummelo (Hirai and Kajiura, 1987; Hirai et al., 1986; Li et al. 2006; Yamamoto and Tominaga, 2003).

Among the various types of DNA analysis, chloroplast (cp) DNA analysis is useful for phylogenic and evolutionary studies. Green et al. (1986) and Yamamoto and Kobayashi (1996) demonstrated the usefulness of cpDNA analysis of Citrus by restriction fragment length polymorphism (RFLP). This is a very time-, labor-, and cost-consuming method but has high reproducibility and reliability. As a recent development of the PCR technique, the cleaved amplified polymorphic sequence (CAPS) using universal primers, which is a simple and reliable method for cpDNA analysis, was developed (Arnold et al., 1991; Taberlet et al., 1991), and important results were obtained for Citrus using this approach (Asadi Abkenar et al., 2004a; Nicolosi et al, 2000); however, the materials used in these studies were mainly major accessions, although many different accessions are grown in various citrus cultivation areas.

Therefore, in the present study, various types of local citrus, especially mandarin accessions whose DNA profile has not been well clarified, were collected from the Ryukyu Islands of Japan, Yunnan and Guangxi of China, and West Sumatra of Indonesia to elucidate their diversity of cpDNA. Here we report the polymorphism of cpDNA revealed by CAPS analysis of citrus and discuss their phylogenic relationships.

\section{Materials and Methods}

Ninety-seven Citrus, four Fortunella, and two Poncirus accessions were used in this study (Table 1). The sources of the materials are shown in Table 1. Total DNA was extracted from leaves using Isoplant II (Nippon Gene, Tokyo, Japan).

Three regions of cpDNA, rbcL-ORF106, trnL-trnF, and $t r n \mathrm{~F}$-trn $\mathrm{Vr}$ (Table 2), were amplified using universal primers (Arnold et al., 1991; Dumolin-Lapegue et al., 1997; Taberlet et al., 1991). The PCR reaction mixture of $15 \mu \mathrm{L}$ consisted of $10 \mathrm{ng}$ template DNA, $10 \mathrm{pmol}$ of each primer, $2 \times$ Ampdirect Plus, and 0.375 units of Nova Taq Hot Start DNA polymerase (Shimadzu, Kyoto, Japan). PCR reactions were performed in a PC320 (Astec, Fukuoka, Japan) thermal cycler programmed as follows: initial heating at $94^{\circ} \mathrm{C}$ for $1 \mathrm{~min}, 35$ cycles of denaturing at $94^{\circ} \mathrm{C}$ for $1 \mathrm{~min}, 1 \mathrm{~min}$ at the annealing temperature (Table 2), extension at $72^{\circ} \mathrm{C}$ for $2 \mathrm{~min}$, and a final extension of $10 \mathrm{~min}$ at $72^{\circ} \mathrm{C}$. Aliquots of amplified products were digested with 5 units of the following enzymes: $r b c \mathrm{~L}-\mathrm{ORF} 106$ products were digested with HinfI or HhaI, trnL-trnF products were digested with Sau3AI, and trnF-trn $\mathrm{Vr}$ products were digested with TaqI. These combinations of primers and enzymes demonstrated polymorphisms in citrus (Asadi Abkenar, personal communication; Asadi Abkenar et al., 2004a; Urasaki et al., 2005). The digested products were electrophoresed on 1.5\% agarose gels (Seakem GTG Agarose; Takara Bio, Shiga, Japan), and detected by staining with Mupid-Stain Eye (Advance, Tokyo, Japan).

\section{Results}

Each primer pair generated a single monomorphic fragment, and the sizes were 3100,450 , and $3000 \mathrm{bp}$ for $r b c \mathrm{~L}-\mathrm{ORF} 106$, trnL-trnF, and trnF-trn $\mathrm{Vr}$, respectively. Polymorphic bands were obtained in all four primer/ enzyme combinations. Citrus accessions used in this study were classified into three types in the combinations of $r b c \mathrm{~L}-\mathrm{ORF} 106 /$ HinfI or HhaI and trnF-trn Vr/TaqI and two types in the combination of $\operatorname{trnL}-\operatorname{trnF} / S a u 3 \mathrm{AI}$ (Fig. 1, Table 3). In each primer/enzyme combination, accessions were classified as follows (Tables 3 and 4):

rbcL-ORF106/HinfI (type a: papeda, C. aurantifolia, C. medica, C. limon, C. grandis, C. natsudaidai, sour and sweet oranges, and their relatives, except for C. tankan, C. sphaerocarpa, C. nobilis (Kunenbo), C. keraji, C. oto, C. tarogayo, C. suavissima, mandarins of Yunnan and Guangxi, China, miscellaneous of Yunnan, China, C. madurensis, and Fortunella), (type b: C.jambhiri, C.tankan, C. ichangensis, C.junos, mandarins, and its relatives except for C.nobilis (Kunenbo), C.keraji, C. oto, C.tarogayo, and C. suavissima, mandarins of Kagoshima, Japan, Guangxi, Zejiang, Yunnan and Guangdong, China, and mandarins of West Sumatra, Indonesia), and (type c: Poncirus);

$r b c$ L-ORF106/HhaI (type a: the accessions belonged "type a" in rbcL-ORF106/HinfI, C.jambhiri, C. tankan, C. ichangensis, C.junos, C.tachibana, C.sunki, C. reshni, C. depressa, mandarins of Kagoshima, Japan and Yunnan and Guangxi, China), (type b: C.nobilis (King), C.unshiu, C. reticulata, C.genshokan, C. clementina, C. succosa, C. suhuensis, C.tardiferax, C. erythora, C.kinokuni, C.oleocarpa, C. leiocarpa, mandarins of Kagoshima, Japan, Guangxi, Zejiang, Yunnan and Guangdong, China, and mandarins of West Sumatra, Indonesia), and (type c: Poncirus);

trnL-trnF/Sau3AI (type a: the accessions belonged "type a" in rbcL-ORF106/HinfI, C.ichangensis, C.junos, C.tachibana, C. depressa (four accessions), Poncirus) and (type b: C.jambhiri, C. tankan, C. nobilis (King), C.unshiu, C. reticulata, C.genshokan, C. clementina, C. succosa, C. suhuensis, C.tardiferax, C. erythora, C. kinokuni, C. sunki, C. reshni, C. depressa (ten accessions), C. oleocarpa, C. leiocarpa, mandarins of Kagoshima, Japan, Guangxi, Zejiang, Yunnan and Guangdong, China, and mandarins of West Sumatra, Indonesia);

$\operatorname{trn} \mathrm{F}-\operatorname{trn} \mathrm{Vr} / \operatorname{Taq} \mathrm{I}$ (type a: the accessions belonged "type a" in rbcL-ORF106/HinfI except for C.latipes, C. ichangensis, C.junos, the accessions belonged "type b" in trnL-trnF/Sau3AI, Poncirus), (type b: C. latipes), 
Table 1. The materials used in this study, their distribution and source, and type of cpDNA.

\begin{tabular}{|c|c|c|c|c|c|c|}
\hline \multirow{2}{*}{ No. } & \multirow{2}{*}{ Common name } & \multicolumn{2}{|c|}{ Latin name } & \multirow{2}{*}{ Distribution } & \multirow{2}{*}{ Source ${ }^{x}$} & \multirow{2}{*}{$\begin{array}{l}\text { Type of } \\
\text { cpDNA }^{w}\end{array}$} \\
\hline & & Tanaka system ${ }^{2}$ & Swingle system ${ }^{y}$ & & & \\
\hline \multicolumn{7}{|c|}{ Archicitrus } \\
\hline \multicolumn{7}{|c|}{ Papeda } \\
\hline & Melanesian papeda & Citrus macroptera Mont. & C. macroptera Mont. & Southeastern Asia & 1 & 1 \\
\hline 2 & Mauritius papeda & C. hystrix DC. & C. hystrix DC. & Southeastern Asia & 1 & 1 \\
\hline 3 & Khasi papeda & C. latipes (Swingle) Tanaka & C. latipes (Swingle) Tanaka & India & 1 & 2 \\
\hline \multicolumn{7}{|c|}{ Lime and its relatives } \\
\hline 4 & Mexican lime & C. aurantifolia (Cristm.) Swingle & C. aurantifolia (Cristm.) Swingle & East Indian Archipelago & 1 & 1 \\
\hline \multicolumn{7}{|c|}{ Citron and its relatives } \\
\hline & Marubusshukan & C. medica $\mathrm{L}$. & C. medica $\mathrm{L}$. & India & 1 & 1 \\
\hline 6 & Xiangyuan (Jianshui) & C. medica $\mathrm{L}$. & C. medica $\mathrm{L}$. & Yunnan, China & 2 & 1 \\
\hline 7 & Xiangyuan (Shiping) & C. medica $\mathrm{L}$. & C. medica $\mathrm{L}$. & Yunnan, China & 2 & 1 \\
\hline 8 & Allen Eureka & C. limon (L.) Burm. f. & C. limon (L.) Burm. f. & India & 1 & 1 \\
\hline 9 & Rough Lemon & C.jambhiri Lush. & C. limon relative & India & 1 & 5 \\
\hline \multicolumn{7}{|c|}{ Pummelo and its relatives } \\
\hline & Benimadoka & C. grandis (L.) Osbeck & C. grandis (L.) Osbeck & Japan & 1 & 1 \\
\hline & Chandler & C. grandis (L.) Osbeck & C.grandis (L.) Osbeck & U. S. A. & 1 & 1 \\
\hline & Pomelo Ratu & C. grandis (L.) Osbeck & C. grandis (L.) Osbeck & Indonesia & 3 & 1 \\
\hline 13 & Pomelo Raja & C. grandis (L.) Osbeck & C. grandis (L.) Osbeck & Indonesia & 3 & 1 \\
\hline & Beniamanatsu & C. natsudaidai Hayata & C. grandis hybrid & Japan & 1 & 1 \\
\hline \multicolumn{7}{|c|}{ Sour and sweet oranges and their relatives } \\
\hline 15 & Sour orange 'Kabusu' & C. aurantium $\mathrm{L}$. & C. aurantium $\mathrm{L}$. & Japan & 1 & 1 \\
\hline & Chinotto & C. myrtifolia Rafin. & C. aurantium relative & Europe & 1 & 1 \\
\hline 17 & Bergamot & C. bergamia Risso et Poit. & C. aurantium relative & Europe & 1 & 1 \\
\hline 18 & Rokugatsumikan & C. rokugatsu hort. ex Yu. Tanaka & C. aurantium hybrid & Kyushu, Japan & 4 & 1 \\
\hline 19 & Sweet orange 'Hamlin' & C. sinensis (L.) Osbeck & C. sinensis (L.) Osbeck & U. S. A. & 1 & 1 \\
\hline & Sweet orange 'Jemsri Jali' & C. sinensis (L.) Osbeck & C. sinensis (L.) Osbeck & Indonesia & 3 & 1 \\
\hline & Tankan ‘Tarumizu 1 gou’ & C. tankan Hayata & C. sinensis hybrid & Guangdong, China & 1 & 5 \\
\hline \multicolumn{7}{|c|}{ Metacitrus } \\
\hline Ichang & g papeda, Yuzu and their relatives & & & & & \\
\hline & Ichang papeda & C. ichangensis Swingle & C. ichangensis Swingle & Yunnan, China & 1 & 3 \\
\hline & Yuzu & C. junos Siebold. ex Tanaka & C. ichangensis hybrid & China and Japan & 1 & 3 \\
\hline & Kabosu & C. sphaerocarpa hort. ex Tanaka & C.junos hybrid & Oita, Japan & 1 & 1 \\
\hline Mand & arin and its relatives & & & & & \\
\hline & Kunenbo & C. nobilis Lour. & C. reticulata Blanco & Indo-China & 1 & 1 \\
\hline & King & C. nobilis Lour. & C. reticulata Blanco & Indo-China & 1 & 6 \\
\hline 27 & Shagan & C. nobilis Lour. & C. reticulata Blanco & China & 5 & 6 \\
\hline 28 & Satsuma mandarin 'Original strain' & C. unshiu Marcow. & C. reticulata Blanco & Kagoshima, Japan & 1 & 6 \\
\hline 29 & Wenzhougan & C. unshiu Marcow. & C. reticulata Blanco & Guangxi, China & 6 & 6 \\
\hline & Keraji & C. keraji hort. ex Tanaka & C. reticulata Blanco & Kikaijima, Japan & 4 & 1 \\
\hline & Kikai mikan & C. keraji hort. ex Tanaka & C. reticulata Blanco & Kikaijima, Japan & 4 & 1 \\
\hline 32 & Kabuchi & C. keraji hort. ex Tanaka & C. reticulata Blanco & Okinawa, Japan & 4 & 1 \\
\hline & Oto & C. oto hort. ex Tanaka & C. reticulata Blanco & Okinawa, Japan & 4 & 1 \\
\hline 34 & Tarogayo & C. tarogayo hort. ex Tanaka & C. reticulata Blanco & Okinawa, Japan & 4 & 1 \\
\hline & Unju & C. tarogayo hort. ex Tanaka & C. reticulata Blanco & Okinawa, Japan & 4 & 1 \\
\hline 36 & Ponkan 'Yoshida ponkan' & C. reticulata Blanco & C. reticulata Blanco & Japan & 1 & 6 \\
\hline 37 & Genshokan & C. genshokan hort. ex Tanaka & C. reticulata Blanco & Taiwan & 1 & 6 \\
\hline 38 & Clementine & C. clementina hort. ex Tanaka & C. reticulata Blanco & Algeria & 1 & 6 \\
\hline 39 & Bendizao & C. succosa hort. ex Tanaka & C. reticulata Blanco & Zejiang, China & 5 & 6 \\
\hline 40 & Sihuigan & C. suhuiensis hort. ex Tanaka & C. reticulata Blanco & China & 5 & 6 \\
\hline & Manju & C. tardiferax hort. ex Tanaka & C. reticulata Blanco & Zejiang, China & 5 & 6 \\
\hline 42 & Ougan & C. suavissima hort. ex Tanaka & C. reticulata Blanco & Zejiang, China & 5 & 1 \\
\hline 43 & Tachibana & C. tachibana (Makino) Tanaka & C. tachibana (Makino) Tanaka & Japan & 1 & 4 \\
\hline 44 & Tachibana 'Okinawa strain' & C. tachibana (Makino) Tanaka & C. tachibana (Makino) Tanaka & Okinawa, Japan & 4 & 4 \\
\hline 45 & Zhuhongju & C. erythrora hort. ex Tanaka & C. reticulata Blanco & China & 5 & 6 \\
\hline 46 & Kinokuni 'Sakurajima komikan' & C. kinokuni hort. ex Tanaka & C. reticulata Blanco & Japan & 1 & 6 \\
\hline & Nanfengmiju & C. kinokuni hort. ex Tanaka & C. reticulata Blanco & Jiangxi, China & 5 & 6 \\
\hline & Nanfengmiju & C. kinokuni hort. ex Tanaka & C. reticulata Blanco & Guangxi, China & 6 & 6 \\
\hline & Miju & C. sp. & C. reticulata Blanco & Guangxi, China & 6 & 6 \\
\hline 50 & Nianju & C. oleocarpa hort. ex Tanaka & C. reticulata Blanco & Guangdong, China & 5 & 6 \\
\hline 51 & Sunki & C. sunki (Hayata) hort. ex Tanaka & C. reticulata Blanco & Guangdong, China & 1 & 5 \\
\hline 52 & Suanju & C. sunki (Hayata) hort. ex Tanaka & C. reticulata Blanco & Guangdong, China & 5 & 5 \\
\hline 53 & Cleopatra & C. reshni hort. ex Tanaka & C. reticulata Blanco & India & 1 & 5 \\
\hline 54 & Shiikuwasha 'Katsuyama kuganii' & C. depressa Hayata & C. tachibana relative & Okinawa, Japan & 1 & 4 \\
\hline 55 & Shiikuwasha 'Ogimi kuganii' & C. depressa Hayata & C. tachibana relative & Okinawa, Japan & 4 & 4 \\
\hline
\end{tabular}


Table 1. Continued

\begin{tabular}{|c|c|c|c|c|c|c|}
\hline \multirow{2}{*}{ No. } & \multirow{2}{*}{ Common name } & \multicolumn{2}{|c|}{ Latin name } & \multirow{2}{*}{ Distribution } & \multirow{2}{*}{ Source $^{x}$} & \multirow{2}{*}{$\begin{array}{l}\text { Type of } \\
\text { cpDNA }^{w}\end{array}$} \\
\hline & & Tanaka system $^{2}$ & Swingle system ${ }^{y}$ & & & \\
\hline 56 & Shiikuwasha 'Izumi kuganii' & C. depressa Hayata & C. tachibana relative & Okinawa, Japan & 4 & 4 \\
\hline 57 & Shiikuwasha 'Few seeds strain' & C. depressa Hayata & C. tachibana relative & Okinawa, Japan & 4 & 4 \\
\hline 58 & Shiikuwasha 'Okitsu strain' & C. depressa Hayata & C. tachibana relative & Okinawa, Japan & 1 & 5 \\
\hline 59 & Shiikuwasha 'Kabishi' & C. depressa Hayata & C. tachibana relative & Okinawa, Japan & 1 & 5 \\
\hline 60 & Shiikuwasha 'Ishikunibu' & C. depressa Hayata & C. tachibana relative & Okinawa, Japan & 4 & 5 \\
\hline 61 & Shiikuwasha 'Shiikunin (Ama)' & C. depressa Hayata & C. tachibana relative & Tokunoshima, Japan & 4 & 5 \\
\hline 62 & Shiikuwasha 'Shiikunin (Kara)' & C. depressa Hayata & C. tachibana relative & Tokunoshima, Japan & 4 & 5 \\
\hline 63 & Shiikuwasha 'Shiikuribu (Yakomo)' & C. depressa Hayata & C. tachibana relative & Okinoerabujima, Japan & 4 & 5 \\
\hline 64 & Shiikuwasha 'Shiikuribu (Kamishiro) & 'C. depressa Hayata & C. tachibana relative & Okinoerabujima, Japan & 4 & 5 \\
\hline 65 & Shiikuwasha 'Shiikuribu (Masana)' & C. depressa Hayata & C. tachibana relative & Okinoerabujima, Japan & 4 & 5 \\
\hline 66 & Shiikuwasha 'Shiikuribu (Amata)' & C. depressa Hayata & C. tachibana relative & Okinoerabujima, Japan & 4 & 5 \\
\hline 67 & Shiikuwasha 'Shiikuribu (Saodsu)' & C. depressa Hayata & C. tachibana relative & Okinoerabujima, Japan & 4 & 5 \\
\hline 68 & Koji & C. leiocarpa hort. ex Tanaka & C. reticulata Blanco & Japan & 1 & 6 \\
\hline 69 & Shimamikan (Nagashima) & C. sp. & C. reticulata Blanco & Kagoshima, Japan & 1 & 6 \\
\hline 70 & Shimamikan (Amami Oshima) & C. sp. & C. reticulata Blanco & Kagoshima, Japan & 4 & 6 \\
\hline 71 & Kuroshimamikan (Yakushima) & C. sp. & C. reticulata Blanco & Kagoshima, Japan & 4 & 6 \\
\hline 72 & Kuroshimamikan (Kuroshima) & C. sp. & C. reticulata Blanco & Kagoshima, Japan & 4 & 5 \\
\hline 73 & Shiyueju & C. sp. & C. reticulata Blanco & Zejiang, China & 5 & 6 \\
\hline 74 & Gaojintou & C. sp. & C. reticulata Blanco & Yunnan, China & 2 & 1 \\
\hline 75 & Huangguo (Jianshui) & C. sp. & C. reticulata Blanco & Yunnan, China & 2 & 1 \\
\hline 76 & Huangguo (near Jianshui) & C. sp. & C. reticulata Blanco & Yunnan, China & 2 & 6 \\
\hline 77 & Huangguo (Wenshan) & C. sp. & C. reticulata Blanco & Yunnan, China & 2 & 1 \\
\hline 78 & Huangguo (Mile) & C. sp. & C. reticulata Blanco & Yunnan, China & 2 & 1 \\
\hline 79 & Baiju (near Jianshui) & C. sp. & C. reticulata Blanco & Yunnan, China & 2 & 6 \\
\hline 80 & Baiju (Jianshui) & C. sp. & C. reticulata Blanco & Yunnan, China & 2 & 6 \\
\hline 81 & Fengdongju & C. sp. & C. reticulata Blanco & Yunnan, China & 2 & 6 \\
\hline 82 & Suanjuzi & C. sp. & C. reticulata Blanco & Yunnan, China & 2 & 5 \\
\hline & Tangfangju & C. sp. & C. reticulata Blanco & Yunnan, China & 2 & 6 \\
\hline 84 & Gonggan & C. sp. & C. reticulata Blanco & Guangxi, China & 6 & 6 \\
\hline 85 & Huapigan & C. sp. & C. reticulata Blanco & Guangxi, China & 6 & 1 \\
\hline 86 & Chuaxingan & C. sp. & C. reticulata Blanco & Guangxi, China & 6 & 6 \\
\hline & Yeju & C. sp. & C. reticulata Blanco & Guangxi, China & 6 & 5 \\
\hline 88 & Ningmingju & C. sp. & C. reticulata Blanco & Guangxi, China & 6 & 6 \\
\hline 89 & Sihuimiju & C. sp. & C. reticulata Blanco & Guangdong, China & 7 & 6 \\
\hline & Shaju & C. sp. & C. reticulata Blanco & Guangxi, China & 6 & 6 \\
\hline & Jeruk Brastagi & C. sp. & C. reticulata Blanco & West Sumatra, Indonesia & 3 & 6 \\
\hline & Jeruk Keprok & C. sp. & C. reticulata Blanco & West Sumatra, Indonesia & 3 & 6 \\
\hline & Jeruk Siem & C. sp. & C. reticulata Blanco & West Sumatra, Indonesia & 3 & 6 \\
\hline & Limau Talang Babungo & C. sp. & C. reticulata Blanco & West Sumatra, Indonesia & 3 & 6 \\
\hline & Crifta & C. sp. & C. reticulata Blanco & West Sumatra, Indonesia & 3 & 6 \\
\hline \multicolumn{7}{|c|}{ Miscellaneous } \\
\hline & Suanganzi & C. sp. & C. sp. & Yunnan, China & 2 & 1 \\
\hline \multicolumn{7}{|c|}{ Kumquat relatives } \\
\hline & Calamondin & C. madurensis Lour. & C. reticulata relative & China & 1 & 1 \\
\hline \multicolumn{7}{|c|}{ Kumquat } \\
\hline \multicolumn{7}{|c|}{ Protocitrus } \\
\hline & Kinzu & $\begin{array}{l}\text { Fortunella hindsii (Champ. ex Benth.) } \\
\text { Swingle }\end{array}$ & $\begin{array}{l}\text { F. hindsii (Champ. ex Benth.) } \\
\text { Swingle }\end{array}$ & China & 1 & 1 \\
\hline \multicolumn{7}{|c|}{ Eufortunella } \\
\hline & Oval kumquat & F. margarita (Lour.) Swingle & F. margarita (Lour.) Swingle & China & 1 & 1 \\
\hline 100 & Round kumquat & F. japonica (Thumb.) Swingle & F. japonica (Thumb.) Swingle & China & 1 & 1 \\
\hline 101 & Meiwa kumquat & F. crassifolia Swingle & $F$. sp. & China & 1 & 1 \\
\hline \multicolumn{7}{|c|}{ Trifoliate orange } \\
\hline 102 & Rubidoux & Poncirus trifoliata (L.) Raf. & P. trifoliata (L.) Raf. & China & 1 & 7 \\
\hline 103 & Flying Dragon & P. trifoliata (L.) Raf. & P. trifoliata (L.) Raf. & China & 1 & 7 \\
\hline
\end{tabular}

z Latin name by Tanaka's system $(1969,1977)$.

y Latin name by Swingle and Reece (1967).

${ }^{x}$ 1: Faculty of Agriculture, Kagoshima University, 2: Exploration in Yunnan, China, 3: Exploration in West Sumatra, Indonesia, 4: Farmer's orchard in Ryukyu islands, Japan. 5: Citrus Research Institute, Chinese Academy of Agricultural Sciences, 6: Exploration in Guangxi, China, 7: Exploration in Guangdong, China.

w See Table 4. 
Table 2. Pairs of cpDNA primers used for PCR amplification in this study.

\begin{tabular}{llcc}
\hline \hline Primer & \multicolumn{1}{c}{ Sequence } & Tm $\left({ }^{\circ} \mathrm{C}\right)$ & Reference \\
\hline$r b c \mathrm{~L}$ & 5'-ATGTCACCACAAACAGAAACTAAAGCAAGT-3' & 55 & Arnold et al., 1991 \\
ORF106 & 5'-ACTACAGATCTCATACTACCCC-3' & & \\
$t r n \mathrm{~L}$ & 5'-GGTTCAAGTCCCTCTATCCC-3' & 55 & Taberlet et al., 1991 \\
$t r n \mathrm{~F}$ & 5'-ATTTGAACTGGTGACACGAG-3' & & \\
$t r n \mathrm{~F}$ & 5'-CTCGTGTCACCAGTTCAAAT-3' & 57.5 & Dumolin-Lapegue et al., 1997 \\
$t r n \mathrm{Vr}$ & 5'-CCGAGAAGGTCTACGGTTCG-3' & & \\
\hline
\end{tabular}

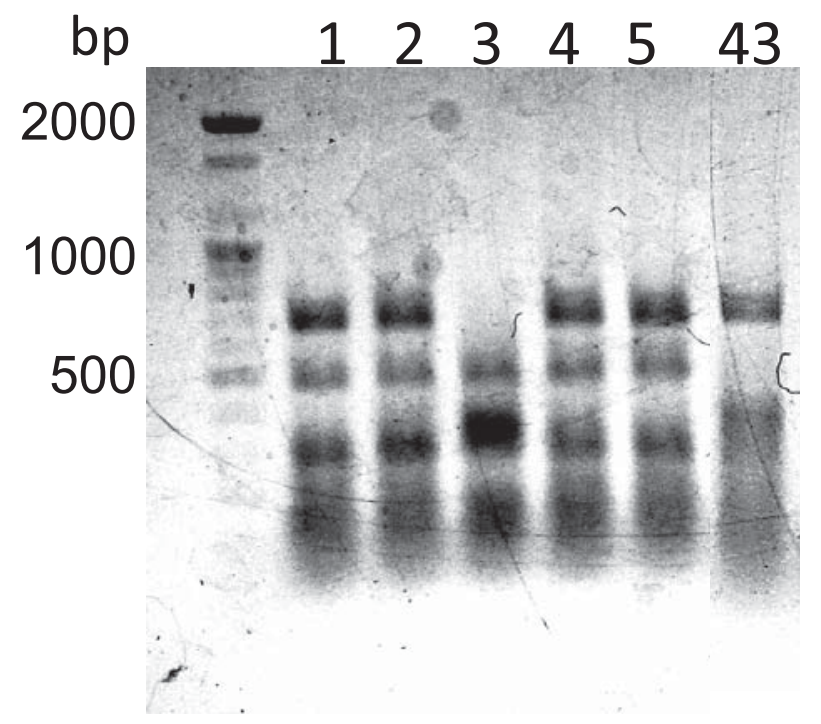

Fig. 1. Restriction pattern obtained after digestion of $t r n \mathrm{~F}-t r n \mathrm{Vr}$ amplified products with TaqI. 1: C. macroptera (type a), 2: C. hystrix (type a), 3: C. latipes (type b), 4: C. aurantifolia (type a), 5: C. medica (type a), 43: C. tachibana (type c).

Table 3. Polymorphic bands that appeared in each primer and enzyme combination.

\begin{tabular}{llcc}
\hline \hline Primers & Enzyme & $\begin{array}{c}\text { Polymorphic band } \\
(\mathrm{bp})^{2}\end{array}$ & Type \\
\hline rbcL-ORF106 & HinfI & 900 & $\mathrm{a}$ \\
& & 600 & $\mathrm{~b}$ \\
& & 950 & $\mathrm{c}$ \\
& HhaI & 1200,600 & $\mathrm{a}$ \\
& & 1200,900 & $\mathrm{~b}$ \\
$\operatorname{trn} \mathrm{L}-\mathrm{trn \textrm {F }}$ & Sau3AI & 4250,600 & $\mathrm{c}$ \\
& & 250,200 & $\mathrm{a}$ \\
$\operatorname{trn} \mathrm{F}-\mathrm{trn} \mathrm{Vr}$ & TaqI & 700,500 & $\mathrm{~b}$ \\
& & 500,400 & $\mathrm{a}$ \\
& & 700,400 & $\mathrm{c}$ \\
\hline
\end{tabular}

${ }^{\mathrm{z}}$ Common bands are excluded.

and (type c: C. tachibana, C. depressa (four accessions)).

All accessions were classified into seven types based on the results of polymorphic bands in all primer/enzyme combinations (Tables 1 and 4) shown as follows:

Type 1 (38 accessions): C. macroptera, C. hystrix, C.aurantifolia, C.medica, C.limon, C. grandis,
C. aurantium,
C. myrtifolia,
C. bergamia,

C. rokugatsu, C. sinensis, C. sphaerocarpa, C. nobilis (Kunenbo), C.keraji, C. oto, C.tarogayo, C. suavissima, mandarins of Yunnan and Guangxi (four and one accessions, respectively), China, miscellaneous of Yunnan, China, C. madurensis, and Fortunella;

Type 2 (one accession): C. latipes;

Type 3 (two accessions): C. ichangensis and C.junos;

Type 4 (six accessions): C. tachibana and C.depressa (four accessions);

Type 5 (18 accessions): C. jambhiri, C. tankan, C. sunki, C. reshni, C. depressa (ten accessions), mandarins of Kagoshima, Japan and Yunnan and Guangxi, China (each one accession);

Type 6 (36 accessions): C.nobilis (King), C. unshiu, C. reticulata, C. genshokan, C.clementina, C. succosa, C. suhuensis, C. tardiferax, C. erythora, C. kinokuni, C. oleocarpa, C. leiocarpa, mandarins of Kagoshima, Japan (three accessions), Guangxi, Zejiang, Yunnan and Guangdong (four, one, five and one accessions, respectively), China, and mandarins of West Sumatra, Indonesia;

Type 7 (two accessions): Poncirus.

\section{Discussion}

The combinations of primers and restriction enzymes used in the present CAPS analysis were established in previous studies that analyzed citrus cpDNA (Asadi Abkenar, personal communication; Asadi Abkenar et al., 2004a; Urasaki et al., 2005). The sizes of the amplified products and the digested fragments in the present and previous studies were identical; however, small bands were not detected well in our results because MupidStain Eye (Advance, Tokyo, Japan) was used as the staining reagent. Mupid-Stain Eye is not hazardous but its resolution is not enough at low molecular range compared with ethidium bromide. Although we could not determine the accurate size of small bands and showed only clear polymorphic bands in Table 3, each type in all combinations of primer and restriction enzyme could be distinguished easily.

In the three basic species of Citrus, C. medica, and C. grandis were not distinguished from each other and both belonged to type 1 in the present study, although previous CAPS analyses using many probes and restriction enzymes (Asadi Abkenar et al., 2004a; Nicolosi et al., 2000) reported a difference of cpDNA 
Table 4. Types of cpDNA of accessions used in this study.

\begin{tabular}{|c|c|c|c|c|c|}
\hline \multirow{2}{*}{ Type } & \multicolumn{2}{|c|}{$r b c \mathrm{~L}-\mathrm{ORF} 106^{\mathrm{z}}$} & \multirow{2}{*}{$\begin{array}{c}t r n \mathrm{~L}-t r n \mathrm{~F} \\
\text { Sau3AI }\end{array}$} & \multirow{2}{*}{$\begin{array}{c}\text { trnF-trn } \mathrm{Vr} \\
\text { Taq } \mathrm{I}\end{array}$} & \multirow{2}{*}{ Accession $^{\mathrm{x}}$} \\
\hline & $\operatorname{Hinfl}^{\mathrm{y}}$ & HhaI & & & \\
\hline 1 & $\mathrm{a}^{\mathrm{w}}$ & $\mathrm{a}$ & a & $\mathrm{a}$ & $\begin{array}{l}1245678101112131415161718192024253031323334 \\
3542747577788596979899100101\end{array}$ \\
\hline 2 & a & $\mathrm{a}$ & a & $\mathrm{b}$ & 3 \\
\hline 3 & $\mathrm{~b}$ & a & a & $\mathrm{a}$ & 2223 \\
\hline 4 & $\mathrm{~b}$ & a & a & $\mathrm{c}$ & 434454555657 \\
\hline 5 & $\mathrm{~b}$ & a & $\mathrm{b}$ & a & 92151525358596061626364656667728287 \\
\hline 6 & $\mathrm{~b}$ & $\mathrm{~b}$ & $\mathrm{~b}$ & a & $\begin{array}{l}26272829363738394041454647484950686970717376 \\
7980818384 \quad 868889909192939495\end{array}$ \\
\hline 7 & $\mathrm{c}$ & $\mathrm{c}$ & a & a & 102103 \\
\hline
\end{tabular}

z Primers.

${ }^{y}$ Restriction enzyme.

${ }^{x}$ See Table 1 .

${ }^{w}$ Each type is shown in Table 3.

between them. On the other hand, mandarins (C. reticulata) showed considerable diversity in their banding patterns and belonged to types 1, 4, 5, and 6 .

C. aurantium, C. sinensis, and C.limon belonged to the same type as $C$.grandis, which is consistent with previous studies (Araújo et al., 2003; Asadi Abkenar et al., 2004a; Tshering Penjor et al., 2010). This result indicates that C. medica or C. grandis was the ancestral female parent of hybrid accessions belonging to type 1 because cpDNA was maternally inherited (Asadi Abkenar et al., 2004b). Japanese mandarins such as C. keraji, C. oto, and C. tarogayo were considered to be derived from C. nobilis (Kunenbo) and to possess the same type as cpDNA of C. sinensis (Yamamoto et al., 2010, 2011). Some of the Chinese mandarins cultivated in Yunnan and Guangxi belonging to type 1 may also be progeny of $C$. sinensis.

Among the papeda accessions, C.macroptera and C. hystrix, and C.latipes belonged to types 1 and 2, respectively; The resemblance of cpDNA of those species to that of $C$. grandis was reported by Nicolosi et al. (2000) and Asadi Abkenar et al. (2004a). On the other hand, cpDNA of $C$. ichangensis was different from both $C$.grandis/C.medica and mandarin. The unique taxonomic form of $C$. ichangensis was also reported by cpDNA analysis of Cheng et al. (2005). C.junos possessed the same type of cpDNA as C.ichangensis. This result supports the concept of genetic relatedness between the two species (Hirai and Kajiura, 1987; Yamamoto et al., 2007).

The cpDNA of mandarins could be classified into three types: types 4,5 , and 6 . Type 4 consists of C. tachibana and C. depressa, mandarins native to Japan. Since this type of cpDNA was not found in mandarins originating from other areas and other Citrus species, these Japanese mandarins, C. tachibana and C. depressa, are considered to be differentiated from other Citrus species. Some Japanese, Chinese, and Indian mandarins belonged to type 5. C. sunki, C. reshni, and C. depressa are small-fruit mandarins mainly used as rootstock. Yeju, a wild mandarin found in China, also belonged to type 5. C. depressa belonged to both type 4 and type 5 . The cpDNA divergence of this species was also reported by Urasaki et al. (2005). These results seemed to indicate a polyphyletic origin of $C$.depressa. This divergence was discovered in C.depressa of Okinawa but not in that of Amami islands (Tokunoshima and Okinoerabujima) because all C.depreesa of Amami islands showed type 5 cpDNA. Various accessions with characteristic morphological traits are cultivated in Okinawa (Kinjo, 2007). The diversity of C. depressa in Okinawa is probably higher than that of the Amami islands based on these results. Froelicher et al. (2011) reported that the mitochondrial (mt) DNA of C. sunki, C. reshni, C. depressa, and C. jambhiri was distinct from that of other mandarins such as C. reticulata, C. unshiu, and C.clementina. This result completely agrees with that of the present study using cpDNA analyses and provides evidence of the differentiation of the cytoplasmic genome in mandarins. Although C. tankan was possibly derived from the cross-combination of C. reticulata and C. sinensis (Yamada, 1994), the types of cpDNA of all three species were different, and thus this hypothesis is rejected by our results. Mandarins such as C.unshiu, C.reticulata, C.clementina, and others whose fruits are consumed fresh or processed generally belonged to type 6. Many mandarins from various regions belonged to this group. Almost all local mandarin accessions cultivated in Yunnan and Guangxi in China and those in Indonesia showed cpDNA of type 6 . Mandarins of type 6, which were collected in Japan, are considered to have arisen from mandarins in other areas because indigenous mandarins (Tanaka, 1936) are type 4 or 5 . On the other hand, the relationship between the collected region and the type of cpDNA was ambiguous in Chinese mandarin. Type 6 is predominant but some mandarins of type 5 were discovered in Guanxi, Yunnan, and Guangdong.

Fortunella resembles Citrus in terms of its general morphological traits, although some of its characteristics 
such as the number of ovules in each locule and the flowering period differ from those of Citrus. Fortunella was not distinguished from Citrus since all the fragment patterns of Fortunella were the same as those of type 1, which includes $C$. grandis, $C$. medica, and some others (Type 1) in the present study. This result agrees with those of previous studies (Bayer et al., 2009; Froelicher et al., 2011; Nicolosi et al., 2000; Yamamoto et al., 1993), which showed no significant differences between Fortunella and Citrus.

Although Poncirus is cross-compatible with Citrus, its various characteristics, deciduous, flowering period, and trifoliate leaves, differ from those of Citrus. The genetic distance between Poncirus and Citrus has already been reported in studies using cp and mtDNA analyses (Bayer et al., 2009; Froelicher et al., 2011; Jung et al., 2005; Yamamoto et al., 1993). In this study, Poncirus belonged to Type 7, which is consistent with the results of the above-mentioned studies.

Simple sequence repeat (SSR) (Kitajima, personal communication), inter-simple sequence repeat (ISSR) (Yamamoto et al., 2010), and sequence-related amplified polymorphism (SRAP) (Yamamoto et al., 2012) analyses revealed that some accessions collected in Japan, China, and Indonesia showed identical DNA profiles. The accessions which were not distinguished on the basis of these DNA analyses are the following: (C. unshiu, No. 28 and 29), (C.tarogayo, No. 34 and 35), (C. kinokuni, No. 46, 47, and 48), (C.depressa, No. 61 and 65), (C. depressa, No. 62-64 and 67), (Chinese mandarin Huangguo, No. 75, 77, and 78), (Chinese mandarin Baiju, No. 79 and 80), and (Indonesian mandarin, No. 91-94). These probably arose from bud sports or nucellar seedlings and belonged to the same type of cpDNA in this study.

The differentiation of the chloroplast genome of citrus is assumed on the basis of the present cpDNA data. Poncirus and Citrus/Fortunella were differentiated from an ancestral species. Among types 1 to 6 , type 1 is considered to be an ancestral type of cpDNA because several genera and species, including papeda, which is probably the most primitive form of Citrus (Tanaka, 1969), belonged to this type. Both type 2 and type 3 were derived from type 1 . In our hypothesis, C. latipes seemed not to be one of the most primitive species (Tanaka, 1969). An ancestral species of mandarin probably also derived from type 1, and then the mandarins native to Japan (type 4: C.tachibana and C. depressa) differentiated. In terms of the main differentiation route, it seems that type 5 (C. sunki, C. reshni, and C. depressa) arose first and was followed by type 6 (C.reticulata, C. unshiu, C. clementina, and so on), which developed from type 5 . From this point of view, mandarins belonging to type 6 possessed the most differentiated cpDNA compared with that of C. grandis/C. medica.

In the present study, we could reveal the diversity of
cpDNA of Citrus and its related genera using more than 100 accessions, including various local Japanese, Chinese, and Indonesian mandarins, by means of CAPS analysis. We demonstrated that cpDNA of mandarin could be distinguished from pummelo and citron among the three basic Citrus species. Furthermore, divergence of cpDNA within the mandarin was clarified and the differentiation of mandarins native to Japan was elucidated. Recent advances in DNA sequencing techniques have allowed the extensive use of short DNA fragment sequences in the study of phylogenetic relationships. A large body of useful information on Citrus phylogeny was obtained from the sequencing of chloroplast genomes (Araújo et al., 2003; Bayer et al., 2009; Jung et al., 2005; Tshering Penjor et al., 2010). Studies like ours that use many accessions are necessary to obtain further information on citrus phylogeny.

\section{Acknowledgements}

We thank Prof. Ryoji Matsumoto and Dr. Asad Asadi Abkenar of the Faculty of Agriculture, Saga University, for providing valuable advice.

\section{Literature Cited}

Araújo, E. F., L. P. Queiroz and M. A. Machado. 2003. What is Citrus? Taxonomic implications from a study of cp-DNA evolution in the tribe Citreae (Rutaceae subfamily Aurantioideae). Org. Divers. Evol. 3: 55-62.

Arnold, M. L., C. M. Bucker and J. J. Robinson. 1991. Pollenmediated introgression and hybrid speciation in Louisiana irises. Proc. Natl. Acad. Sci. USA 88: 1398-1402.

Asadi Abkenar, A., S. Isshiki and Y. Tashiro. 2004a. Phylogenic relationships in the "true citrus fruit trees" revealed by PCRRFLP analysis of cpDNA. Sci. Hortic. 102: 233-242.

Asadi Abkenar, A., S. Isshiki and Y. Tashiro. 2004b. Maternal inheritance of chloroplast DNA in intergeneric sexual hybrids of "true citrus fruit trees" revealed by PCR-RFLP analysis. J. Hort. Sci. Biotechnol. 79: 360-363.

Barkley, N. A., M. L. Roose, R. R. Kruegar and C. T. Federici. 2006. Assessing genetic diversity and population structure in a citrus germplasm collection utilizing simple sequence repeat markers (SSRs). Theor. Apple. Genet. 112: 1519-1531.

Bayer, R. J., D. J. Mabberley, C. Morton, C. H. Miller, I. K. Sharma, B. E. Pfeil, S. Rich, R. Hitchcock and S. Sykes. 2009. A molecular phylogeny of the orange subfamily (Rutaceae: Aurantioideae) using nine cpDNA sequences. Am. J. Bot. 96: 668-685.

Cheng, Y., M. C. de Vincente, H. Meng, W. Guo, N. Tao and X. Deng. 2005. A set of primers for analyzing chloroplast DNA diversity in Citrus and related genera. Tree Physiol. 25: 661672.

Dumolin-Lapegue, S., M. H. Pemonge and R. J. Petit. 1997. An enlarged set of consensus primers for the study of organelle DNA in plants. Mol. Ecol. 6: 393-397.

Federici, C. T., D. Q. Fang, R. W. Scora and M. L. Roose. 1998. Phylogenic relationships within the genus Citrus (Rutaceae) and related genera as revealed by RFLP and RAPD analysis. Theor. Appl. Genet. 96: 812-822.

Froelicher, Y., W. Mouhaya, J-B. Bassene, G. Costantino, M. Kamiri, F. Luro, R. Morillon and P. Ollitrault. 2011. New universal mitochondrial PCR markers reveal new information 
on maternal citrus phylogeny. Tree Genet. Genomes 7: 49-61.

Green, R. M., A. Vardi and E. Galun. 1986. The plastome of Citrus. Physical map, variation among Citrus cultivars and species and comparison with related genera. Theor. Appl. Genet. 72: 170-177.

Hirai, M. and I. Kajiura. 1987. Genetic analysis of leaf isozymes in citrus. Japan. J. Breed. 37: 377-388.

Hirai, M., I. Kozaki and I. Kajiura. 1986. Isozyme analysis and phylogenic relationship of citrus. Japan. J. Breed. 36: $377-$ 389.

Kinjo, H. 2007. Acid citrus "Shiikuwasha" in Ryukyu island. Studia Citrologica 17: 137-148 (In Japanese).

Jung, Y. H., H. M. Kwon, S. H. Kang, J. H. Kang and S. C. Kim. 2005. Investigation of the phylogenetic relationships within the genus Citrus (Rutaceae) and related species in Korea using plastid trnL-trnF sequences. Sci. Hortic. 104: 179-188.

Li, Y. Z., Y. J. Cheng, H. L. Yi and X. X. Deng. 2006. Genetic diversity in mandarin landraces and wild mandarins from China based on nuclear and chloroplast simple sequence repeat markers. J. Hort. Sci. Biotechnol. 81: 371-378.

Nicolosi, E., Z. N. Deng, A. Gentile, S. La Malfa, G. Continella and E. Tribulato. 2000. Citrus phylogeney and genetic origin of important species as investigated by molecular markers. Theor. Appl. Genet. 100: 1155-1166.

Pang, X. M., C. G. Hu and X. X. Deng. 2007. Phylogenic relationships within Citrus and its related genera as inferred from AFLP markers. Genet. Resour. Crop Evol. 54: 429-436.

Swingle, W. T. and P. C. Reece. 1967. The botany of Citrus and its wild relatives. p. 190-430. In: W. Reuther, H. J. Webber and L. D. Batchelor (eds.). The citrus industry, vol. 1. University of California, Berkeley.

Taberlet, P., L. Gielly, G. Pautou and J. Bouvet. 1991. Universal primers for amplification of three non-coding regions of chloroplast DNA. Plant Mol. Biol. 17: 1105-1109.

Tanaka, T. 1936. Citrus studies (In Japanese). Yokendo, Tokyo.

Tanaka, T. 1969. Misunderstanding with regards citrus classification and nomenclature. Bull. Univ. Osaka Pref., Ser. B. 21: 139-145.
Tanaka, T. 1977. Fundamental discussion of citrus classification. Studia Citrologica 14: 1-5.

Tshering Penjor, T. Anai, Y. Nagano, R. Matsumoto and M. Yamamoto. 2010. Phylogenetic relationships of Citrus and its relatives based on $r b c L$ gene sequences. Tree Genet. Genomes 6: 931-939.

Urasaki, N., K. Yoshida, T. Uehara, H. Inoue, S. Onda, H. Kinjyo and S. Kawano. 2005. Single nucleotide polymorphism in shiikuwasha (Citrus depressa Hayata) chloroplast DNA, trnLtrnF. Jpn. J. Trop. Agr. 49: 246-251.

Yamada, Y. 1994. Tankan and Kunenbo. P. 1-6. Japan Fruit Tree Seeding and Clonal Association, Tokyo (In Japanese).

Yamamoto, M. and S. Kobayashi. 1996. Polymorphism of chloroplast DNA in citrus. J. Japan. Soc. Hort. Sci. 65: 291296.

Yamamoto, M. and S. Tominaga. 2003. High chromosomal variability of mandarin (Citrus spp.) revealed by CMA banding. Euphytica 129: 267-274.

Yamamoto, M., A. Asadi Abkenar, R. Matsumoto, H. Nesumi, T. Yoshida, T. Kuniga, T. Kubo and S. Tominaga. 2007. CMA banding patterns of chromosomes in major Citrus species. J. Japan. Soc. Hort. Sci. 76: 36-40.

Yamamoto, M., M. Fukuda, T. Koga, T. Kubo and S. Tominaga. 2010. Examination of the origin of Keraji (Citrus keraji), local citrus of Kikaijima island, Kagoshima prefecture. Hort. Res. (Japan) 9: 7-12 (In Japanese with English abstract).

Yamamoto, M., S. Kobayashi, Y. Nakamura and Y. Yamada. 1993. Phylogenic relationships of citrus revealed by RFLP analysis of mitochondrial and chloroplast DNA. Japan. J. Breed. 43: 355-365.

Yamamoto, M., R. Kouno, T. Nakagawa, T. Usui, T. Kubo and S. Tominaga. 2011. Isozyme and DNA analyses of local Citrus germplasm on Amami Islands, Japan. J. Japan. Soc. Hort. Sci. 80: 268-273.

Yamamoto, M., N. Nasir, T. Ninomiya, T. Kubo and S. Tominaga. 2012. Fruit characteristics, chromosome and DNA profiles of four mandarins (Citrus reticulata Blanco) collected in West Sumatra, Indonesia. South Pacific Studies 32: 59-69. 